\title{
EL TIEMPO EN LA FÍSICA ATÓMICA
}

Las categorías del conocimiento científico expresan conceptualmente las formas comunes $-\mathrm{y}$, por lo tanto, más generales- de existencia de los procesos objetivos. Se forman en el desarrollo de las investigaciones particulares de cada ciencia y se modifican -ensanchándose en amplitud y hondura- a medida que progresa el conocimiento científico. Y este proceso de transformación de las categorías se desenvuelve con mayor rapidez cuando se exploran niveles de la realidad que antes eran desconocidos. En la actualidad, la física indaga justamente el nivel recientemente descubierto de los procesos atómicos, en el cual se han encontrado muchas propiedades que difieren notablemente de las existentes entre los procesos mesocósmicos y macrocósmicos. Sin embargo, las interpretaciones filosoficas de sus resultados no han sido muy profusas y, en su mayor parte, coinciden en su orientación idealista y en una especie de "ortodoxia cuántica" propiciada por algunos físicos tan eminentes como Bohr y Heisenberg. Por ello, es importante realizar un examen crítico y objetivo de las características peculiares que muestra el tiempo en la existencia microcósmica, par pod pr señalar algunos de los elementos necesarios a la integración filosófica de esta categoría en la física atómica. $Y$ esto es lo que aquí nos hemos propuesto hacer.

\section{Conjugación de energía y tiempo}

Como se sabe, Heisenberg hizo el descubrimiento de que la determinación simultánea de la energía y el tiempo tiene un límite de precisión para el caso de las partículas elementales. Esto quiere decir que, mientras más exactamente se determina el instante en que una partícula elemental se encuentra en un cierto lugar, más incierta resulta ser la determinación que se haga entonces de su energía; y, recíprocamente, mientras más precisa sea la determinación de la energía de una partícula atómica, menos exacta será la determinación del momento en que se encuentre en un lugar dado. ${ }^{1}$ Sin embargo, es completamente posible determinar la energía de una partícula con el grado de precisión que se quiera; y, también, es enteramente posible determinar el instante en que pasa por un lugar con toda la precisión que se necesita. ${ }^{2}$ Lo

1 Entre la incertidumbre de la energía $\Delta E$, y la incertidumbre del tiempo, $\Delta t$, se cumple la relación: $\Delta E: \Delta t \equiv \frac{h}{4 \pi}$.

2 Esto es que, conforme a la propia relación de incertidumbre, cuando se determina la energía de una partícula con toda la precisión requerida, esta determinación no perturba a la magnitud de dicha energía y, por lo tanto, al medir inmediatamente después [64] 
único que no se puede conseguir es determinar el tiempo y la energía de la misma partícula con una precisión infinita. ${ }^{3}$ Desde el punto de vista lógico, tenemos que la relación simultánea entre los conceptos de "energía precisamente determinada" y de "instante precisamente determinado" para una y la misma partícula elemental, se expresa por medio de un juicio de incompatibilidad. Por consiguiente, existen tres posibilidades: a) determinar con precisión la energía, en detrimento de la determinación precisa del tiempo; $b$ ) determinar con precisión el tiempo, a costa de la determinación precisa de la energía; y, c) determinar simultáneamente la energía y el tiempo con la precisión permitida por el límite de la relación de incertidumbre.

Ahora bien, en la interpretación arbitraria que se hace con arreglo al llamado "principio de complementariedad", la relación de incertidumbre es convertida subjetivamente en una exclusión radical entre las dos primeras posibilidades señaladas antes, con la consiguiente consideración absurda de la inexistencia de la tercera posibilidad. A la vez, la interpretación complementarista sostiene a todo trance los conceptos de energía y tiempo establecidos en la física clásica -atribuyéndoles un carácter inmutable y absoluto ${ }^{4}$ - para poder concluir luego que, como no es posible determinarlos separadamente y con ese carácter en los procesos atómicos, no se pueden conocer objetivamente las propiedades de las partículas elementales. De esta manera, se intenta convertir a las propiedades fundamentales de la existencia en el nivel atómico, en categorías subjetivas y decididamente irracionales. Sin embargo, aun dejando a un lado la "conversión" complementarista, parece ser conceptualmente imposible determinar el tiempo y la energía de una partícula elemental en un mismo lugar, con una precisión que exceda al límite establecido por la relación de incertidumbre. Pero, por otro lado, tenemos que en una de las ecuaciones primordiales de la mecánica cuántica, la energía $E$ de una partícula elemental está determinada justamente por la constante cuántica universal $h$ y la frecuencia de su movimiento ondulatorio $v \rightarrow 0$ sea el número de crestas de onda que pasan por un punto en la unidad de tiem-

de nuevo la energía de la partícula se encuentra exactamente el mismo valor anterior. Lo que sí habrá cambiado con la determinación de la energía es el tiempo de la propia partícula. Y lo mismo ocurre respecto al tiempo, que no se altera al ser determinado con la mayor exactitud.

3 El límite finito de la precisión está determinado con toda exactitud por el segundo miembro de la desigualdad: $\frac{h}{4 \pi}$.

4 En rigor, ni siquiera en la física clásica se puede sostener ahora el concepto de tiempo absoluto -en el sentido de ser independiente de las otras propiedades objetivas-, ya que eso exigiría la transmisión instantánea de señales, lo cual es imposible por el hecho de que no existen transferencias instantáneas de energía. Tampoco se puede considerar actualmente la separación estricta de tiempo y energía en la física clásica. Y, en cuanto a la inmutabilidad de los conceptos científicos, basta señalar que el avance del conocimiento los enriquece constantemente $y$, por ende, los hace cambiar de continuo; y esto ocurre igualmente, desde luego, con los conceptos básicos que son las categorías. 
po-, conforme a la relación: $E=h v$. Entonces, resulta que el cumplimiento de esta ecuación impone la necesidad de tomar en cuenta y operar simultáneamente con la energía y con una propiedad temporal -como lo es la frecuencia que, dimensionalmente, es el inverso del tiempo- de una y la misma partícula elemental. Más aún, la ecuación requiere ineludiblemente la consideración de valores precisos de ambas magnitudes; lo cual, de acuerdo con la relación de incertidumbre, es justamente imposible hacer con precisión respecto a la energía y al tiempo. Así se plantea una paradoja insostenible: por un lado, la imposibilidad de hacer la determinación precisa y simultánea de la energía y el tiempo; $y$, por otra parte, la necesidad imposible de esquivar, de hacer la determinación simultánea y precisa de la energía y la frecuencia - propiedad temporal también - de una partícula atómica. En consecuencia, es conceptualmente posible y experimentalmente necesario determinar a la vez, para una y la misma partícula, la magnitud de su energía y de una de sus propiedades temporales.

La misma irreductibilidad de la oposición indicada plantea claramente la urgencia de superar esta paradoja, resolviéndola dialécticamente. Para esto, tenemos que considerar la energía de una partícula elemental como otra forma en que se manifiesta su frecuencia u oscilación ondulatoria; y, a la vez, tenemos que reconocer en la frecuencia de su movimiento ondulatorio otra manera en la cual se muestra su energía. Así, el tiempo tiene que ser considerado conjuntamente como energía y oscilación; o, en otras palabras, se tiene que reconocer a la energía y a la frecuencia como dos formas, distintas pero inseparables, a través de las cuales se manifiesta ese modo de existencia de las partículas atómicas que constituyen lo que llamamos sus propiedades temporales. ${ }^{5}$ Entonces, podemos expresar su relación lógica en función de un juicio de inclusión. Respecto a las partículas elementales, el tiempo se puede manifestar como ascilación, o como energía; o bien, como oscilación y energía a la vez. De esta manera quedan satisfechas simultáneamente la relación de incertidumbre y la ecuación que conjuga la frecuencia del movimiento ondulatorio con la energía; lo cual resulta posible, entre otras razones, por la coincidencia de la constante cuántica $h$ en ambas ecuaciones.

\section{Tiempo y espacio}

En la física cuántica se ha encontrado que las partículas elementales tienen una naturaleza integrada por dos aspectos contradictorios e indisolubles: su constitución corpuscular y su movimiento ondulatorio. El aspecto corpuscular, caracterizado por la energía y la cantidad de movimiento, representa una masa de dimensiones cuánticas $y$, por lo tanto, muestra su existencia discon-

5 Ya se ha demostrado antes, en el dominio de la teoría de la relatividad, esta unión entre el tiempo y la energía. 
tinua. Mientras que el movimiento ondulatorio, caracterizado por la frecuencia y la longitud de onda, representa su extensión continua y homogénea. La conjugación inseparable entre ambos aspectos se muestra en el hecho de que la cantidad de movimiento y la longitud de onda son dos maneras diferentes en que se exhiben las propiedades espaciales; ${ }^{6}$ en tanto que la energía y la frecuencia son, por su parte, dos modos distintos en que se manifiestan las propiedades temporales. ${ }^{7}$ Es más, esta conjugación se encuentra determinada con precisión en las ecuaciones fundamentales de la mecánica cuántica, que enlazan por parejas a las cuatro magnitudes mencionadas en las seis relaciones posibles entre ellas. ${ }^{8}$ Por otro lado, se ha comprobado que cuando se determinan con precisión la energía y la cantidad de movimiento de una partícula elemental $\longrightarrow$ sea, las características de su aspecto corpuscular- lo que se consigue es que la partícula se haga difusa a través de una enorme extensión espacial, perdiéndose casi por entero su aspecto corpuscular. Análogamente, cuando se determina con exactitud la posición de una partícula elemental en un tiempo dado - esto es, las características de su movimiento ondulatoriolo que se obtiene es una extremada imprecisión de su estado de movimiento. Entonces, tenemos que la determinación del estado de movimiento de la partícula elemental —es decir, de sus condiciones dinámicas- y su localización espaciotemporal -o sea, la determinación de sus condiciones de movimiento- son dos planos de la realidad que no es posible enfocar con precisión al mismo tiempo. Más aún, cuando se enfoca uno de estos planos con la mayor exactitud, lo que se observa con nitidez es justamente el otro plano; y viceversa. Por lo tanto, no se trata en rigor de una exclusión mutua, sino de una conexión estrecha e indisoluble entre ambos enfoques. Así tenemos, primero, la conjugación de tiempo y espacio en la doble forma de energía-cantidad de movimiento y de frecuencia-longitud de onda; $y$, luego, el enlace entre estas dos conjugaciones espaciotemporales. De este modo, queda destacada la interdependencia múltiple entre el tiempo y el espacio de las partículas elementales y la relación recíproca entre su localización espaciotemporal y la especificación de su estado dinámico.

Ahora bien, debido a que entre las partículas elementales se encuentran los fotones o cuantos de luz y a que las otras partículas se mueven con velocidades comparables a la de la luz, es indispensable considerar las propiedades descubiertas por la teoría de la relatividad que se cumplen también en los procesos atómicos. Desde luego, tenemos con ello otra confirmación de que

- Véase mi artículo "La categoría de espacio en la física atómica", Diánoia, III, 1957; sec. 5, págs. 105-111.

7 Véase la primera sección del presente artículo.

$8 E=p c ; v=\frac{c}{\lambda} ; p=\frac{h}{\lambda} ; \lambda=\frac{h c}{E} ; E=h v ; p=\frac{h v}{c}$. Siendo: $E$, la energía; $p$, la cantidad de movimiento; $c$, la velocidad de la luz; $v$, la frecuencia; $\lambda$ la longitud de onda; y $h$, la constante de Planck. 
las propiedades atómicas no se pueden determinar aisladamente en el espacio, sino que tienen que ser determinadas simultáneamente con el tiempo; y, lo que es más, tenemos que las propiedades locales del espacio-tiempo dependen de la distribución de masa y energía en la región correspondiente. ${ }^{9}$ Como ya lo hemos indicado antes, en los procesos atómicos se amplía el mutuo condicionamiento entre el espacio-tiempo y la distribución estática de energía y masa, con la inclusión de la influencia recíproca que se ejerce entre el estado cinemático y el estado dinámico de las partículas elementales. Por otra parte, conforme a las consideraciones relativistas, debemos distinguir dos aspectos en el tiempo: las propiedades temporales de los procesos que se desarrollan unos cerca de otros en el espacio, o tiempo local; y las propiedades de los procesos que se encuentran considerablemente alejados en el espacio; o tiempo universal. Como se advierte, las propiedades del tiempo universal están inextricablemente unidas a las propiedades del espacio considerado en toda su extensión. Lo que se hace comúnmente es tomar las propiedades locales del tiempo dentro de una región espacial restringida -cuyas propiedades métricas son constantes- $y$, luego, dichas propiedades son extendidas espacialmente al universo entero. Igualmente, para determinar las propiedades espaciales lo que se hace es tomar un subconjunto del espaciotiempo - en el cual se considera como constante el tiempo- para generalizar después estas propiedades en el tiempo, haciéndolas abarcar también la totalidad del universo. ${ }^{10}$ Con todo, está claro que la extensión del tiempo local al universo implica necesariamente la consideración de la variación métrica del espacio; y, a su vez, la extensión del espacio local trae consigo la inclusión de la variación del tiempo. Entonces, para establecer una determinación rigurosa de estas propiedades, se plantea ineludiblemente la necesidad de investigar las variaciones temporales y espaciales que se producen en el espacio-tiempo local en el cual se desarrollan los procesos atómicos. En fin, la espacialización del tiempo - - , si se prefiere, la temporalización del espacioconduce logicamente a sintetizar el tiempo y el espacio como componentes de la existencia, en el sentido de que los intervalos temporales son simplemente muchísimo mayores que los intervalos espaciales. Así, podemos establecer que un intervalo mide un centímetro mientras otro dura un segundo, con la diferencia de que este segundo intervalo puede ser hasta treinta mil millones de veces mayor que el primero. ${ }^{11} \mathrm{Y}$, por lo demás, ambos intervalos se pueden convertir recíprocamente.

9 En el espacio-tiempo relativista se tienen dos vectores tetradimensionales en los cuales se encuentran conjugados el espacio y el tiempo $(x, y, z, t)$ y la cantidad de movimiento y la energía con signo negativo $\left(p_{s}, p_{y}, p_{z},-E\right)$.

$10 \mathrm{O}$ sea, que se sigue el procedimiento utilizado por Newton para establecer sus conceptos de tiempo y espacio.

11 Esta relación de magnitud entre el espacio y el tiempo es la misma que existe entre la masa y la energía, tal como se expresa en la ecuación de Einstein: $E=m c$. 


\section{Cuantización del tiempo}

Un rasgo común de las dos nuevas físicas - la teoría de la relatividad y la mecánica cuántica - lo constituye el descubrimiento de límites finitos para ciertas propiedades fundamentales. En efecto, tanto en los procesos macrocósmicos como en los procesos microcósmicos -que constituyen los dos extremos de la experiencia científica - se ha determinado la existencia de valores críticos que no pueden ser traspasados. En la teoría de la relatividad, la propiedad singularizada es la velocidad y su valor crítico es $c$, la velocidad de la luz. En la mecánica cuántica, la propiedad destacada es el cambio en la acción y su valor crítico es $h$, la constante universal de Planck. Por consiguiente, la velocidad a que se transmite la energía tiene un límite máximo extremadamente grande, pero finito $\left(c=2.99776 \times 10^{10} \mathrm{~cm} / \mathrm{seg}\right)$. Y, a su vez, los cambios en la acción tienen un límite mínimo sumamente pequeño, pero finito también $\left(h=6.6237 \times 10^{-27} \mathrm{erg} \cdot \mathrm{seg}\right)$. De esta manera, ha quedado en claro que la energía no se transmite nunca de manera instantánea, sino que su transmisión toma siempre un cierto tiempo que, cuando menos, es el correspondiente a la velocidad de la energía luminosa. Además, también sucede que la energía sólo se transfiere en dosis que dependen siempre de múltiplos enteros de una cantidad pequeñísima -el cuanto- sin que sea posible la transferencia de una cantidad de energía menor que cierto valor indiviso. Ahora bien, tiene gran importancia señalar que en ambos valores críticos figura el tiempo como uno de los elementos integrantes de la propiedad física que ha quedado limitada cuantitativamente. ${ }^{12}$ Esto hace destacar indudablemente al tiempo como una de las propiedades primordiales de la existencia. Y, a la vez, significa que el tiempo no puede ser subdividido interminablemente, sino que está formado por momentos que tienen una duración sumamente pequeña, pero no carecen de duración. ${ }^{13}$ Dicho de otro modo, el tiempo tiene características cuánticas - $\mathrm{y}$, por lo tanto, discontinuas- respecto a la transmisión y a la transferencia de energía.

Dentro del átomo, los electrones se mueven siempre a lo largo de las órbitas cuánticas, cuyas distancias al núcleo atómico forman una serie de valores discretos; ${ }^{14}$ y, a la vez, las longitudes de estas trayectorias son múltiplos enteros de la longitud de onda de los propios electrones. A cada una de estas órbitas cuánticas le corresponde una energía de magnitud definida y, por consiguiente, una frecuencia u oscilación temporal también finita. Así, existe una rigurosa discontinuidad en el tiempo que tarda un electrón en recorrer las distintas trayectorias orbitales. Por otro lado, tenemos que el paso

12 La velocidad es el cambio espacial en el tiempo y la acción es el producto de la energía por el tiempo.

$13 \mathrm{El}$ orden de magnitud del cuanto de tiempo es de 10-23 segundos.

14 La distancia mínima, en el caso más simple del átomo de hidrógeno, es de $0.527 \times 10^{-8}$ centímetros. 
del electrón de una órbita a otra, ya sea por absorción o por emisión de energía, se produce de manera brusca, como un "salto cuántico". Esta energía es absorbida o emitida de ordinario en forma de radiación y su magnitud está conectada con su frecuencia, o sea, con el inverso del tiempo. Por lo tanto, en la transferencia mutua de energía radiante entre el átomo y el exterior, el tiempo muestra igualmente su carácter cuántico. En lo que se refiere al interior del átomo, lo podemos representar como una sucesión de transcursos temporales continuos - correspondientes a las revoluciones orbitales posiblesseparados por intervalos en donde nunca se encuentran los electrones, pero que permiten el salto de estos últimos de una órbita a otra. Por ende, el tiempo interno del átomo está constituido por trozos de continuidad separados entre sí y cuyo enlace se establece de manera súbita e intermitente. Estos saltos bruscos de los electrones impiden su representación en un tiempo continuo y tampoco permiten la consideración de una continuidad espacial. Por lo demás, en las órbitas - que son temporalmente continuas- la cantidad de movimiento del electrón queda determinada por su energía y la longitud de la trayectoria se encuentra definida por su frecuencia. O sea, que aquí nos encontramos de nuevo con la conjugación dialéctica entre el espacio, el tiempo, la energía y la cantidad de movimiento de las partículas atómicas.

Fuera del átomo, las trayectorias de las partículas elementales no pueden ser determinadas de una manera unívoca y continua. Es posible establecer con precisión, por ejemplo, la salida de un fotón y su llegada, o sea, su emisión y su absorción; pero, no es posible determinar rigurosamente cuál es la trayectoria que sigue entre su emisión y su absorción. Además, la partícula elemental se manifiesta únicamente cuando reacciona con otras partículas $y$, por consiguiente, sólo se muestra de trecho en trecho, sin que se le pueda atribuir una trayectoria continua. Entonces, la mecánica cuántica se limita a vincular entre sí las diversas manifestaciones sucesivas y discontinuas de la existencia temporal de una partícula, sin poder precisar lo que ocurre en el intervalo intermedio. Esto significa que las propiedades temporales de la partícula elemental se exhiben sólo de una manera intermitente. A la vez, dentro del átomo, tampoco es posible hacer una determinación temporal directa de las partículas elementales. Porque no se puede medir directamente la duración de la revolución orbital de un electrón; es decir, que no es posible determinar el tiempo que transcurre en un espacio definido como es la órbita cuántica. Y, lo que es más, tampoco es posible hacer determinación alguna de la duración de un salto cuántico de una órbita a otra. En cambio, sí se pueden determinar con exactitud las frecuencias de la energía radiante emitida por el átomo como consecuencia de un salto cuántico. Más aún, es posible determinar aproximadamente las características temporales de la órbita, junto con el estado cuántico de la partícula que la recorre. Con todo, parece obligado aceptar la existencia de trayectorias intermitentes para el movimiento de las partículas 
elementales. Y, por lo tanto, tenemos que tomar en cuenta la posibilidad de que el tiempo se manifieste como una discontinuidad cinemática en el nivel atómico de l̦a existencia.

\section{Causalidad cuántica}

El determinismo clásico lo podemos expresar sencillamente de la siguiente manera: Cuando se conocen con precisión las condiciones en que se encuentra un sistema de procesos en un instante cualquiera y, además, se conocen las leyes que gobiernan su comportamiento, entonces, es posible prever rigurosamente las condiciones en que se encontraría ese mismo sistema en cualquier otro instante futuro o pasado. Como se advierte desde luego, es indispensable la conjugación del conocimiento de las leyes naturales con el conocimiento de las condiciones específicas iniciales, para que el determinismo se pueda plantear de manera necesaria y suficiente. Por otro lado, se debe hacer notar que el presente físico nunca es un instante sin dimensión, sino que implica siempre un lapso de cierta duración y, por lo tanto, como las condiciones iniciales no son estrictamente instantáneas, incluyen al menos una breve evolución del sistema considerado. Además, dichas condiciones iniciales deben persistir en el tiempo, aun cuando varíen en su magnitud. Por otra parte, la interacción de todos los procesos existentes impone la exigencia de conocer el estado del universo en un lapso dado, para poder establecer una previsión rigurosa; $y$, como esto es enteramente irrealizable, se recurre a la consideración de sistemas relativamente aislados en donde sea suficiente la aproximación de conocer un número finito de condiciones iniciales. Igualmente, se requiere la ejecución de medidas precisas de las condiciones iniciales $y$, como se sabe, la medición científica es siempre imperfecta; $y$, lo que es más, las mismas previsiones que se pueden establecer a partir de estos datos aproximados también implican cierta imprecisión. Sin embargo, hay que añadir el hecho innegable de que la precisión de las observaciones y el rigor de las previsiones científicas se mejora continuamente. Como consecuencia de todo esto, podemos decir que, en la física clásica, el determinismo constituye el límite al cual converge el conocimiento.

En lo que se refiere a la mecánica cuántica, las relaciones de incertidumbre expresan una frontera insalvable para el conocimiento simultáneo y preciso de algunas parejas de magnitudes físicas que integran las condiciones que caracterizan un sistema de procesos atómicos. Es más, sirviéndonos de una ilustración experimental muy clara, presentada por Louis de Broglie ante el IX Congreso Internacional de Filosofía, podemos mostrar la imposibilidad de determinar con precisión singular el comportamiento de una partícula elemental. Tomemos un "disparador de electrones" -esto es, un artefacto que emite electrones de energía conocida - para bombardear una superficie 
de cristal y frente a ésta colocamos una pantalla fluorescente en la cual se produce una centelluela a la llegada de cada electrón. Entonces, cuando emitimos electrones con lentitud, observamos cómo las centelluelas se suceden en el tiempo y se producen en puntos diversos de la pantalla. Pero es imposible prever exactamente cuál será el punto de la pantalla en donde se producirá en cierto momento la siguiente chispa. Lo único que se puede hacer es calcular la probabilidad de que sea en tal o cual punto de la pantalla en donde se observe la próxima centelluela. Así, las chispas individuales son verdaderamente imprevisibles $y$, en consecuencia, no es posible sostener el determinismo físico en el sentido clásico. ${ }^{15}$ Pero, no obstante, es indudable que sólo veremos aparecer centelluelas en la pantalla cuando se haga funcionar el disparador y que, por otro lado, la densidad de estas chispas dependerá de la frecuencia con que se efectúen los disparos; mientras que, cuando el disparador no funciona, es obvio que tampoco aparece chispa alguna en la pantalla. Por consiguiente, sí podemos decir que el funcionamiento del disparador de electrones es la causa del centelleo en la pantalla, aun cuando no podamos prever exactamente cuál de las centelluelas será la que se produzca al poner en acción el disparador. Entonces, podemos afirmar que en este caso se cumple la relación de causalidad. $O$ sea, dicho en otras palabras, que se cumple rigurosamente el determinismo en su aspecto cualitativo de concatenación entre causas y efectos, a pesar de que no se pueda decir lo mismo acerca del aspecto cuantitativo del determinismo. Esta conclusión sobre el cumplimiento inequívoco de la causalidad se puede generalizar congruentemente a todos los problemas que se presentan en la física atómica. ${ }^{18}$ Lo cual implica que, incluso suponiendo irreductible el indeterminismo de la presente forma de la teoría de los procesos cuánticos, lo cierto es que en estos procesos se cumple estrictamente la causalidad.

\section{Relaciones entre el tiempo y la causalidad}

Con los descubrimientos hechos por Einstein y ror su profundo examen crítico de la simultaneidad, se ha puesto en claro que el tiempo constituye el

15 En cambio, cuando los electrones son disparados con gran rapidez, de modo que el cristal sea alcanzado constantemente por un torrente de electrones, entonces, en cada momento se tendrá un centelleo continuo, cuyas chispas se distribuirán dibujando las figuras conocidas de la difracción. $\mathrm{Y}$, así, podremos anticipar exactamente cuál va a ser el aspecto de la pantalla fluorescente. Por lo tanto, para el conjunto de muchísimos electrones sí existe la previsibilidad rigurosa y se puede afirmar el cumplimiento del determinismo clásico.

16 Este cumplimiento riguroso de la causalidad en los procesos cuánticos es completa- • mente independiente del fruto que puedan tener los trabajos de David Bohm, De Broglie, Vigier, Janossy, Blojintzev, Frenkel, Terletski, Takabayasi y otros, que están orientados hacia la constitución de una interpretación determinista y objetiva de la mecánica cuántica, para superar las graves inconsecuencias en que se encuentra la teoría cuántica "ortodoxa" actual. 
orden de las concatenaciones causales. La relación causal es reducible al orden temporal establecido por lo anterior y lo posterior, porque la causa siempre antecede al efecto y, a su vez, el efecto sigue siempre a la causa. Esto no representa una mera coincidencia, sino que pone de manifiesto la estrecha conexión existente entre el orden temporal y los procesos causales. Como se ha demostrado, el orden es invariante bajo las transformaciones de Lorentz - para el cambio de coordenadas entre dos sistemas que se mueven entre sí- únicamente cuando los sistemas de que se trata pueden ser conectados por señales, ${ }^{17}$ o sea, por cadenas causales. En algunos casos, las transformaciones de Lorentz permiten la inversión del orden temporal entre ciertos procesos; pero, en rigor, esta inversión sólo es posible respecto de aquellos procesos que no pueden ser conectados causalmente. Un proceso $A$ está conectado causalmente con un proceso $B$, cuando: $A$ es causa de $B, \circ B$ es causa de $A$, o existe otro proceso $C$ que es causa común de $A$ y de $B$, o hay un proceso $D$ que es efecto común de $A$ y de $B$; o bien, cuando se tiene una acción causal recíproca entre $A$ y $B$, o cuando ambos se encuentran en conexión mutua de causa y efecto con otro u otros procesos. En todo caso, los procesos que se encuentran en conexión causal son temporalmente colindantes y, entre ellos, distinguimos como causa al antecedente y como efecto al consecuente. De esta manera, definimos la causa mediante el tiempo. Por lo demás, las causas son contemporáneas de sus efectos, al menos parcialmente; esto es, la causa comienza antes que el efecto, pero permanece por lo menos durante una parte del tiempo ocupado por el efecto. Como la concatenación causal se establece mediante la transmisión de energía, resulta que no hay efectos que se produzcan instantáneamente, debido a que la velocidad máxima para la transmisión de energía es la de la luz. Por consiguiente, siempre transcurre cierto tiempo entre la aparición de la causa y la producción del efecto, así sea un lapso pequeñísimo. Además, la repetición de una causa trae consigo la repetición del efecto. Y esta reiteración de una cadena causal tiene idéntica duración; es decir, las mismas causas emplean el mismo tiempo en producir los mismos efectos $\mathrm{y}$, análogamente, causas aproximadamente iguales emplean casi el mismo tiempo en producir efectos muy semejantes. Entonces, cuando una conexión causal es conocida científicamente, se puede prever rigurosamente su repetición cuando se vuelve a presentar la misma causa o se provoca su presentación. Con este conocimiento de las relaciones causales y de la posibilidad de su repetición, el hombre cuenta con uno de los instrumentos más eficaces para intervenir en el curso de los procesos existentes, siempre que consiga provocar o inducir la presentación de las causas. De este modo, y procurando la conveniente intensificación o reducción de las

17 Señal es una discontinuidad en la transmisión de energía que media entre causa $y$ efecto cuando éstos distan. 
condiciones en que se producen las cadenas causales, es como el hombre ejerce su dominio sobre el mundo.

Ahora bien, el hacer una previsión con base en el conocimiento de una conexión causal, entraña ineludiblemente referir al futuro el pasado conocido. O sea, es tanto como traspasar lo ocurrido en un cierto intervalo de tiempo ya transcurrido a otro intervalo de tiempo por transcurrir. $\mathrm{Y}$ esto implica necesariamente la consideración de que el tiempo es homogéneo y congruente y de que tiene un sentido único. La homogeneidad del tiempo señala su característica de ser el orden único, común y fundamental en el cual se manifiestan todos los cambios que se desarrollan en los procesos existentes. La congruencia del tiempo significa la posibilidad de trasladar un lapso de un intervalo a otro, manteniendo invariantes sus propiedades y sus resultados; $y$, también, la posibilidad de superponer varios intervalos a modo que coincidan en un solo lapso. ${ }^{18}$ En fin, el sentido único del tiempo indica aquí el hecho de que la reiteración de una cadena causal se produce de la misma manera, sin que sea factible su reversión. Por otra parte, las conexiones causales son irreflexivas, porque su relación no se puede aplicar a un proceso aislado y por sí solo; o sea, que ningún proceso es causa única de sí mismo, ni tampoco es el único efecto de sí mismo. Pero, a la vez, las conexiones causales son reflexivas, puesto que todo proceso es parcialmente causa y efecto de sí mismo; y, lo que es más, siempre es posible considerar un acontecimiento causal como un proceso unitario complejo, en el cual las causas y los efectos constituyen sus elementos integrantes. Igualmente, la relación causal es asimétrica, puesto que cuando un proceso $A$ es causa de otro proceso $B$, entonces, este proceso $B$ es efecto de $A$. Śblo que, también puede ser simétrica una conexión causal, cuando $A$ es causa y efecto de $B$ y, a su vez, $B$ es efecto y causa de $A$. Por otra parte, las cadenas causales son transitivas, ya que: si un proceso $A$ es causa de otro proceso $B$, y este proceso $B$ es por su parte causa de otro $C$, entonces, el proceso $A$ es causa mediata de $C$. Matemáticamente, la causalidad se puede expresar por medio de una función en la cual la variable independiente es la causa y la variable dependiente es el efecto. Más aún, toda función matemática es una expresión abstracta de la causalidad, en la que no se incluye la secuencia temporal; o sea, que se abstrae el intervalo temporal como si no existiera. ${ }^{19}$ Por lo tanto, debido a que todas las leyes físicas se expresan cuantitativamente en forma de funciones matemáticas, tenemos que la causalidad se encuentra contenida necesariamente y sin excepción en las leyes físicas. Sin embargo, debido a su simetría, las funciones matemáticas $-\mathrm{y}$; por ende, las leyes físicas explicitadas por ellas- admiten la inversión. ${ }^{20}$

18 Esta superposición temporal, aceptada ya en la teoría ondulatoria clásica, es análoga a la congruencia de la geometría arquimediana.

19 Debido a esto es que la relación de causalidad no queda caracterizada exhaustivamente por el concepto matemático de relación funcional.

20 Si $y$ es una función de $x$, entonces, $x$ es una función de $y$; $y$, en consecuencia, lo 
Esto significa que las leyes físicas no definen un solo sentido para la causalidad - sino que son indiferentes para ambos sentidos- $y$, por lo tanto, las funciones matemáticas -y las leyes que expresan- representan necesariamente a la causalidad, pero no lo hacen de un modo suficiente. Y su insuficiencia resulta justamente de la abstracción que en ellas se hace de la secuencia temporal. Entonces, aplicando lógicamente la inferencia causal, tenemos que el tiempo posee inherentemente un sentido único y que este sentido es compartido por la relación causal cuando, y sólo cuando, es considerada en su integridad, esto es, incluyendo el orden del tiempo.

Como la velocidad de la transmisión causal está limitada por la velocidad de la energía luminosa, puede ocurrir que un proceso $C$ no sea causa ni efecto de un proceso $D$, cuando la distancia que los separa sea tan grande que la energía no se pueda transmitir oportunamente de $C$ a $D$, ni tampoco de $D$ a $C$. Esto significa que la acción recíproca entre dos procesos -cuya manifestación más conspicua es la relación de causalidad- tiende a cero contemporáneamente, cuando los procesos se alejan indefinidamente entre sí. Para tales parejas de procesos no es posible establecer un orden temporal definido. Por lo tanto, nos encontramos aquí con un hecho recíproco al que hemos apuntado antes. Es decir, que la causalidad tiene inherentemente un orden definido y que este orden es compartido por la sucesión temporal cuando, y sólo cuando, se puede incluir en el tiempo la conexión causal. ${ }^{21}$ Con todo, debido a la acción recíproca existente entre todos los procesos, resulta que los procesos contemporáneos tienen siempre causas comunes mediatas en el pasado -así sean extremadamente remotas-, a pesar de que en el momento considerado no exista directamente conexión causal entre ellos - esto es, que no sean causas ni efectos unos de otros. De este modo, es posible considerar la causalidad universal como una generalización de las causalidades locales, o sea, de las concatenaciones causales existentes dentro de las proximidades permitidas por la velocidad de la transmisión de la energía. Por último, es importante destacar que entre las leyes físicas, unas se refieren explícitamente a la formulación de conexiones causales - como la ley de la conservación y la transformación de la energía que, por cierto, se cumple rigurosamente en los procesos atómicos-, otras expresan definidamente el comportamiento de los procesos físicos sin determinar su sentido - como la mayor parte de las leyes de la mecánica clásica, de la relatividad y de la mecánica cuántica- y otras más indican inequívocamente el sentido en que se desarrollan los procesos sin admitir su reversibilidad -como ocurre con algunas leyes relativas

que era variable independiente -0 causa- se convierte en variable dependiente -0 efecto-, y viceversa.

21 Esto mismo es aplicable en último extremo al orden espacial; ya que un lugar $X$ se encuentra más cerca de $Y$ que de $Z$, cuando una señal luminosa directa -0 sea, la cadena causal más rápida- transmitida de $X$ a $Z$ pasa antes por $Y$. 
al comportamiento de los procesos atómicos en masa-.22 Pero, en todo caso, siempre se encuentran implícitas en toda ley física las conexiones de causalidad, así sea en forma abstracta o incompleta. Más aún, en las leyes físicas nunca se admiten anomalías causales porque, últimamente, lo que en ellas se expresa primordialmente es la regularidad de la existencia objetiva $y$, en este sentido, las causas son indagadas justamente para explicar las irregularidades que se presentan en la regularidad universal.

\section{Dimensionalidad del tiempo}

De acuerdo con la física clásica, el tiempo es monodimensional. Y, conforme a la teoría de la relatividad, además de ser monodimensional, queda definido completamente por una coordenada única en el continuo espaciotiempo; pero es indispensable que no se confunda la dimensión temporal con las dimensiones espaciales del continuo. Así, para determinar un proceso con respecto al tiempo, es necesario y suficiente realizar una sola medida en lá dimensión temporal única. Porque, cuando se consideran hipotéticamente dos o más dimensiones temporales, se pierde la conexión causal y la determinación deja de ser unívoca. Por consiguiente, en la física relativista se confirma la monodimensionalidad del tiempo, aun cuando no se hace distinción alguna entre los dos sentidos de esta dimensión única. ${ }^{23}$. En este aspecto, la representación relativista del tiempo es semejante a la consideración temporal de las ecuaciones de la física clásica. En efecto, como se sabe, las ecuaciones diferenciales de la mecánica son de segundo orden y no tienen primeras derivadas respecto al tiempo. Entonces, si $f(t)$ es una solución para alguna de estas ecuaciones, también es solución $f(-t)$, porque en la doble diferencia-

22 Es interesante traer a cuento lo ocurrido respecto a las interpretaciones sostenidas por Compton y Bohr, respecto a la conexión entre el impacto de un fotón y la emisión de un electrón. Compton consideraba que entre estos dos procesos hay una conexión causal precisa, establecida como consecuencia de las leyes de conservación; mientras que Bohr negaba la existencia de semejante conexión, afirmando que su apariencia causal era simplemente un efecto estadístico. Entonces se efectuó el experimento de Bothe y Geiger, consistente en recibir los electrones emitidos y los fotones desviados en cámaras de ionización adecuadas, en donde se puede registrar su entrada. Si la interpretación de Compton era correcta, la penetración de un electrón y de un fotón en las respectivas cámaras sería simultánea; pero, si Bohr tenía razón, las penetraciones ocurrirían al azar y sin concierto. Pues bien, el experimento demostró que las penetraciones simultáneas ocurren con una frecuencia mucho mayor de la que podría esperarse conforme a las leyes de la probabilidad. Es más, cuando se toman en cuenta los efectos parásitos que es fácil evitar, se consigue que las penetraciones simultáneas se conviertan en regla. Así se comprobó experimentalmente, una vez más, la existencia de conexiones causales rigurosas entre los procesos atómicos individuales.

23 Por supuesto, el hecho de que el sentido del tiempo no esté incluido en las ecuaciones clásicas y relativistas, no es una prueba en contra de la existencia de dicho sentido, ya que una interpretación científica puede abstraer algunas propiedades de los procesos, sin que esto signifique que tales propiedades no existan $-y$ tal cosa ocurre frecuentemente con el tiempo o, al menos, con su sentido. 
ción se presenta una doble multiplicación por $\frac{d(-t)}{d t}=-1$, lo cual hace que el resultado sea el mismo en ambos casos. Esto significa que para cada proceso conocido siempre es posible, en general, encontrar una representación inversa. ${ }^{24}$ Tal posibilidad de inversión deja indefinido el sentido del tiempo, pero sí define, en cambio, la dimensión temporal única - ya que solamente puede haber inversión de sentido estrictamente respecto a una y la misma dirección- y, también, pone de manifiesto el orden simétrico de la relación de causalidad, que es invariante para el cambio de sentido del tiempo.

Ahora bien, este carácter monodimensional del tiempo se muestra de manera todavía más acusada en la física atómica. En efecto, la ecuación de Schroedinger que gobierna el desarrollo temporal de la función $\Psi$ es de primer orden respecto a la variable $t y$, por lo tanto, distingue nítidamente un sentido del tiempo de su opuesto. La función $\Psi(q)$ suministra una descripción tan completa como es posible del estado de un sistema $y$, cuando este estado cambia en el curso del tiempo, la variable $t$ ingresa como otro argumento de la función. Por ello, Schroedinger ha establecido una ecuación diferencial de esta función $\Psi(q, t)$, que expresa la ley fundamental del cambio en la mecánica cuántica. Entonces, el sentido del tiempo en el cual ocurre el cambio se manifiesta en el signo del argumento $t$. Así, cuando $\Psi(q, t)$ es una solución de la ecuación, entonces $\Psi(q,-t)$ no es solución, y viceversa. Por lo tanto, queda planteado el problema de discriminar entre estas dos funciones, o sea, de tomar inicialmente uno $\mathbf{u}$ otro sentido $(+o-)$ para el transcurso temporal. Pero, como se advierte, una vez hecha la elección del sentido, éste ya no se puede invertir porque la ecuación no lo admite. En consecuencia, la ecuación ofrece una demostración importante de la monodimensionalidad del tiempo en los procesos atómicos. A la vez, aun cuando no precisa unívocamente entre el sentido positivo y el sentido negativo del tiempo, sí establece la exclusión entre ambos y sólo admite uno de ellos. De esta manera, se hace un avance fundamental con respecto a las ecuaciones clásicas y relativistas, que admiten indistintamente los dos sentidos temporales y permiten su inversión mutua, por ser ecuaciones de segundo orden sin primera derivada temporal. Y, también, se consigue otro progreso fundamental, ya que se desecha la idea de la reversibilidad del tiempo cuántico.

\section{Sentido del tiempo}

Los procesos irreversibles -combustión, ruptura, mezcla de líquidos o gases, transferencia de calor, etc. - se caracterizan por la conservación o el

24 Pero esto no indica que para cada proceso exista otro que se desarrolle en el sentido negativo del tiempo. 
incremento de la entropía, ${ }^{25}$ esto es, por la tendencia hacia la desaparición de las diferencias existentes entre los diversos estados. Su expresión matemática adopta la forma de una función monótona no-decreciente, en la cual el incremento de la variable temporal produce siempre un aumento en la magnitud de la entropía, o bien, su persistencia en la magnitud anterior, pero nunca su decrecimiento. Por lo tanto, los procesos irreversibles sí definen un sentido para el tiempo, que es precisamente aquel en que la entropía no decrece, o sea el sentido positivo. La primera ley de la termodinámica ${ }^{26}$ establece que en todos los intercambios existe una magnitud, llamada energía, cuyo valor permanece constante. Por su parte, la segunda ley de la termodinámica establece la existencia de otra magnitud, denominada entropía, ${ }^{27}$ cuyo valor permanece constante en algunos cambios y en otros aumenta. ${ }^{28}$ Entonces, tomando en cuenta la conjugación existente entre la energía y el tiempo, podemos señalar también la existencia de una correlación inseparable entre el tiempo y la entropía. De este modo, la ley de la conservación de la energía viene a ser otra expresión del orden asimétrico de la causalidad y, por ende, de la monodimensionalidad del tiempo con la admisión de uno u otro de sus sentidos. En cambio, la ley de la conservación y el no-decrecimiento de la entropía representa la definición de un solo sentido para el tiempo. Por consiguiente, es enteramente logico utilizar la segunda ley de la termodinámica como base para fijar un sentido unívoco al tiempo.

Es importante advertir que las leyes termodinámicas indican el sentido del tiempo, pero son ajenas a su cuantificación; es decir, que señalan al flujo del tiempo un sentido unívoco, sin hacer referencia a la métrica de este transcurso. ${ }^{29}$ También se puede tomar como índice una muestra de algún

25 Entropía es una magnitud definida en función de propiedades termodinámicas - como la temperatura, la presión, la energía y el volumen- en la cual no se consideran las propiedades elementales de la mecánica como la posición y la velocidad.

20 Conviene tener presente aquí el requisito de correspondencia entre las teorías fisicas, o sea, la exigencia de que en los procesos atómicos considerados en masa, las propiedades cuánticas deban convertirse por completo en las propiedades termodinámicas.

27 En rigor, el comportamiento termodinámico de un proceso está determinado por una función característica, cuyo conocimiento es suficiente para determinar unívocamente las condiciones de los distintos estados de equilibrio físico y químico en que se puede encontrar dicho proceso. Y la forma de esta función característica depende de las variables independientes consideradas: a) cuando dichas variables son la energía y el volumen, la función característica es la entropía; $b$ ) cuando las variables son el volumen y la temperatura, la función característica es la correspondiente a la energía libre; y c) cuando las variables son la temperatura y la presión, la función característica es el potencial termodinámico dividido entre la temperatura con signo negativo.

28 Como es sabido, la tercera ley de la termodinámica -formulada por Nernst en 1906 y verificada después ampliamente por la experiencia científica- establece que el valor de la entropía tiende a anularse cuando la temperatura de un procesó decrece indefinidamente, y que, para temperaturas finitas, la entropía no puede tener un valor finito. $Y$, como consecuencia de este descubrimiento, se ha derrumbado definitivamente la base de todas las fantásticas especulaciones hechas acerca de la "muerte térmica del universo".

29 En cambio, otras leyes físicas que determinan la métrica del tiempo no definen su sentido. 
elemento radioactivo $\mathrm{y}$, entonces, entre dos momentos $t_{a}$ y $t_{b}$ queda definido como posterior aquel en el cual la cantidad de la muestra sea menor $y$, recíprocamente, es anterior el momento en que la cantidad de la muestra es mayor; con la ventaja de que el lapso transcurrido entre los dos momentos tiene un valor medio que se puede determinar con precisión. ${ }^{30}$ Por lo tanto, en la desintegración radioactiva tenemos un patrón objetivo que nos suministra la medida del tiempo y su sentido. Asimismo, la transmisión de señales - por intermedio de la cual se establecen las cadenas causales- es un proceso ligado con el crecimiento termodinámico de la entropía y, en consecuencia, nos ofrece igualmente otro medio de medir el tiempo tomando en cuenta la irreversibilidad de su sentido. Más aún, incluso dentro de la teoría "ortodoxa" de la mecánica cuántica se considera definido el sentido del tiempo; puesto que, por un lado, se supone que un conjunto de partículas elementales y su entropía no cambian en nada cuando no se les observa $y$, por otra parte, se sostiene que la descripción de estados es indagada en una sucesión de pruebas, lo cual da por resultado la consideración del desarrollo monótono del conjunto con su tendencia a una entropía mayor, prueba tras prueba; con la circunstancia de que el registro de las pruebas muestra cuál prueba fue anterior y cuál fue posterior en ese desenvolvimiento. Por todo esto, podemos establecer que en un proceso cuántico elemental - como el movimiento de un solo electrón- el sentido del tiempo queda determinado unívocamente por su correspondencia con el tiempo de los procesos atómicos en masa.

En cuanto al sujeto que realiza las observaciones, tenemos que el hombre es parte del mundo objetivo y que su memoria es un instrumento de registro que está gobernado por las leyes de la teoría de la información. Y, de acuerdo con estas leyes, el incremento de la información define también el sentido del tiempo. Las experiencias pasadas se encuentran registradas en nuestra memoria, mientras que las experiencias futuras sólo podrán quedar registradas cuando se efectúen y nunca antes. Así, el tiempo subjetivo de nuestra experiencia no es otra cosa que la manifestación de las propiedades temporales de los procesos objetivos, a través del instrumento de registro que es nuestra memoria. Pero, esta manera de definir el sentido del tiempo no es un privilegio humano, ya que todo instrumento de registro - ya sea natural $o$ artificialhace lo mismo. En consecuencia, esta definición del sentido del tiempo surge de la relación entre el instrumento de registro y su medio; y, por la isotropía y la homogeneidad ${ }^{31}$ del universo, esta relación viene a ser la misma para

30 En el caso de los procesos termodinámicos no es posible hacer esta determinación cuantitativa del intervalo temporal en que se desarrollan, porque este lapso varía de una instancia a otra o, más bien, su desenvolvimiento es indiferente a la magnitud del tiempo transcurrido.

31 Por isotropía se entiende que en el universo no existe preferencia alguna por un lugar o una dirección en particular. Homogeneidad significa que la historia del universo es siempre idéntica en su contenido, aunque se le enfoque desde los más diversos lugares. 
todos los instrumentos de registro, incluyendo a la memoria humana. En lo que se refiere a las hipótesis de E. C. G. Stückelberg ${ }^{32}$ y R. P. Feynman, ${ }^{33}$ acerca de que un positrón - esto es, un electrón con carga positiva - puede ser considerado como un electrón que se mueve en sentido contrario al tiempo, ${ }^{34}$ no deja de ser una mera especulación matemática sugerida por el ensayo de cambiar simultáneamente los signos de la carga y del tiempo en las ecuaciones correspondientes al electrón. ${ }^{35}$ Además, con arreglo a semejante hipótesis, se perdería el orden dimensional del tiempo, se invertiría la conexión causal y resultaría que una y la misma partícula existiría en más de un espécimen a la vez. ${ }^{36}$ En fin, con referencia a la posibilidad de escoger inicialmente un sentido para el tiempo en la ecuación de Schroedinger - sentido que ya no se puede invertir luego sin alterar la ecuación- no se debe olvidar que las funciones matemáticas no siempre representan la realidad de una manera suficiente, sino que en ellas se permite muchas veces la abstracción de alguna o algunas propiedades fundamentales de la existencia. Y, en particular, hay que tener en cuenta que la matemática se ha especializado profundamente en la investigación de las propiedades del espacio, ${ }^{37}$ bajo el supuesto abstracto de la constancia del tiempo. En suma, por todo lo expuesto, podemos concluir que el sentido unívoco del tiempo queda definido sin ambiguiedad por algunas de las leyes de los procesos atómicos y que, por otra parte, este sentido único e irreversible es enteramente compatible con todas las demás leyes de la física cuántica, lo mismo que con la totalidad de las leyes de la mecánica clásica y de la teoría de la relatividad.

32 "Remarque à propos de la création de paires de particules en théorie de relativite", Helv. Phys. Acta, 14, 1941; 588-594; "La mécanique du point matériel en théorie de relativité et en théorie des quanta", ibid., 15, 1942, 23-37.

33 "The theory of positrons", Phys. Rev., 76, 1949; 749-759.

34 En tal caso, la carga negativa unitaria del electrón que viaja en sentido temporal opuesto, tiene los mismos efectos teóricos que la carga unitaria que viaja en sentido directo del tiempo.

35 Otra especulación análoga consistiría en formular la hipótesis de que el neutrón fuese tenido como un protón para el cual la carga y el tiempo quedaran anulados.

36 Por ejemplo, en lugar del conocido fenómeno de que un rayo $\gamma$ se transforme en una pareja negatrón-positrón y, luego, el positrón se conjugue con otro negatrón -electrón ordinario con carga negativa - para constituir otro rayo $\gamma$, lo que se tendría así entre ambos rayos $\gamma$ es la existencia de uno y el mismo electrón en tres especímenes distintos: un negatrón con sentido temporal positivo, un negatrón con sentido temporal negativo y otro negatrón con sentido temporal positivo.

37 El fundamental descubrimiento hecho recientemente por Lee Tsung-dao y Yang Chen-ning -por el cual se les otorgó merecidamente el Premio Nobel de Física en 1957de que en la transmutación de las partículas elementales no se cumple el principio de conservación de la paridad, plantea de manera inmediata la necesidad imperiosa de formular una nueva teoría física para explicar los procesos atómicos. A la vez, demuestra que el espació real es asimétrico. Por lo tanto, no habiendo simetría entre la izquierda y la derecha desaparece por entero la posibilidad de sostener, por analogía, que el futuro y el pasado sean simétricos o, lo que es lo mismo, que el tiempo sea reversible. 


\section{Cronología discontinua}

Como es sabido, la topología —o sea la geometría más general- se ocupa del estudio de las transformaciones espaciales que son reversibles, biunívocas y continuas. A resultas de este estudio se determinan las propiedades del espacio que son invariantes a toda clase de distorsiones, con excepción de la ruptura. Pero, tal como sucede en las otras geometrías, en la topología también se hace abstracción del tiempo. No obstante, las investigaciones topologicas permiten la obtención - por estricta analogía - de conclusiones acerca de las propiedades del tiempo, en tanto que éste puede considerarse parcialmente idéntico al orden asimétrico del espacio en una sola dimensión y reducido a un sentido único. Empleando este procedimiento es posible verificar las siguientes propiedades temporales: a) el tiempo transcurre del pasado al futuro; $b$ ) el presente divide localmente al pasado del futuro, y viceversa; c) el pasado nunca retorna, es irrevocable; $d$ ) es posible tener registros del pasado, pero no del futuro; $e$ ) es posible cambiar el futuro, pero no el pasado, porque existe la oportunidad de intervenir de algún modo en el porvenir; f) la intervención en el futuro está condicionada por el pasado; g) el pasado se encuentra determinado, mientras que el futuro está indeterminado, aunque es determinable en principio; $h$ ) el presente es un intervalo que puede hacerse muy pequeño o alargarse mucho, pero siempre es finito y su duración depende de la distancia entre los procesos comparados temporalmente; $i$ ) el presente es el lapso en donde los acontecimientos no son pasados ni futuros y, a la vez, en donde ocurre la coincidencia entre el futuro y el pasado; $y, j$ ) no hay simultaneidad entre todos los lugares, de tal modo que un presente local puede corresponder al futuro en distinto lugar y al pasado en otro lugar más.

Finalmente, debemos acoger aquí la cuestión que ya planteamos en nuestro ensayo sobre el espacio atómico, ${ }^{38}$ de si las propiedades de los procesos cuánticos exigen la construcción de una geometría todavía más general que la topología, en la cual se pueda "romper" el espacio sin perjuicio de que se conserven invariantes algunas de sus propiedades; o sea, dicho de otro modo, de si será menester la formulación de una geometría que estudie las transformaciones discontinuas. Desde luego, considerando las características del tiempo puestas de manifiesto por la física atómica, y en tanto que la geometría estudia indirectamente y de modo abstracto ciertas propiedades temporales, resulta clara la confirmación de la necesidad de realizar esta investigación en torno a la discontinuidad. Por otro lado, tenemos que las rupturas del espacio, las cuales son incompatibles con su reversibilidad, en cambio, son completamente compatibles con la irreversibilidad del tiempo. Además, es posible establecer una correspondencia analítica entre las propie-

38 Véase en Diánoia, III, 1957; sec. 12, págs. 122-123. 
dades espaciales y las temporales, semejante a la dualidad descubierta entre los puntos y las líneas, de la siguiente manera:

Espacio

Monotopía. Una partícula ocupa un solo lugar al mismo tiempo.

Heterotopía. Un lugar es ocupado por una sola partícula al mismo tiempo.

Politopía. ${ }^{40}$ Una partícula ocupa diversos lugares al mismo tiempo.

Homotopía. ${ }^{41}$ Un lugar es ocupado por varias partículas al mismo tiempo.

Cinetopia. Una partícula ocupa diversos lugares en distintos tiempos.

Isotopia. Un lugar es ocupado por varias partículas en distintos tiempos.

Anisotopía. Varias partículas ocupan diversos lugares en distintos tiempos.
Tiempo

Monocronismo. ${ }^{\text {a9 }}$ Una partícula se encuentra un solo tiempo en el mismo lugar.

Heterocronismo. A un tiempo se encuentra una sola partícula en un mismo lugar.

Policronismo. Una partícula se encuentra en distintos tiempos en el mismo lugar.

Homocronismo. ${ }^{42}$ A un tiempo se encuentran varias partículas en el mismo lugar.

Cinecronismo. Una partícula se encuentra en distintos tiempos en diversos lugares.

Isocromismo. A un tiempo se encuentran varias partículas en diversos lugares.

Anisocronismo. Varias partículas se encuentran en distintos tiempos en diversos lugares.

Entonces, lo que se requiere es la elaboración de una teoría matemática de las transformaciones irreversibles y discontinuas, que ya no será una geometría del tipo de las actuales, sino una representación rigurosa del espaciotiempo. Es decir, que se necesita indagar matemáticamente las propiedades del espacio sin hacer abstracción del tiempo; o sea, incluyendo explícitamente las propiedades temporales en su integridad. Y tal teoría podrá ser denominada, tal vez, cronotópica.

\section{Tiempo atómico}

Tomando como base todo lo examinado anteriormente, podemos apuntar las modalidades asumidas por el tiempo como categoría de la física atómica. Por principio de cuentas, tenemos que la relación temporal es una de las

39 Propiedad atómica del tiempo.

40 Propiedad atómica del espacio.

41 Propiedad atómica del espacio.

42 Propiedad atómica del tiempo. 
formas fundamentales en que se manifiesta la existencia de los procesos microcósmicos. ${ }^{43}$ Sólo que el tiempo no es algo independiente de los procesos, ni constituye tampoco una especie de flujo abstracto en el cual transcurriesen los cambios de las partículas elementales. En realidad, el tiempo es el conjunto de las propiedades temporales inherentes a los procesos atómicos; esto es, que los procesos microcósmicos no existen en el tiempo, sino que su existencia es temporal. Y, lo que es más, el carácter común que tienen las propiedades temporales destaca de modo definido la pertenencia inesquivable de cada una de las partículas elementales a su medio y, en consecuencia, el intercambio activo entre todas ellas. Por otro lado, el tiempo atómico tiene dos aspectos diferentes aunque inseparables, que son la energía y la oscilación. Cada uno de estos aspectos temporales se conjuga con sendas formas del espacio atómico, la energía con la cantidad de movimiento y la frecuencia con la longitud de onda; y, también, estas dos conjugaciones espacio-temporales se enlazan entre sí. De esta manera, se establece una temporalización del espacio que entraña irremediablemente la espacialización del tiempo.

Otra de las propiedades del tiempo atómico es su transcurso continuo, el cual se encuentra aparejado con el carácter cuántico $-\mathrm{y}$, por consiguiente, discontinuo- de la transmisión y la transferencia de la energía. En el interior del átomo, el tiempo se muestra como una sucesión de transcursos temporales continuos - las revoluciones orbitales de los electrones- separados por intervalos discontinuos que sólo permiten el paso de manera brusca e intermitente. Fuera del átomo, las partículas elementales siguen trayectorias discontinuas y únicamente se manifiestan de trecho en trecho. En cambio, en su movimiento ondulatorio, las partículas elementales exhiben su continuidad y su persistencia, tanto temporal como espacialmente. Por otra parte, el tiempo constituye el orden de las conexiones causales, hasta el punto de que las causas son definidas mediante el tiempo y las conexiones causales mismas se establecen a través de la transmisión de energía - que es otra propiedad temporal. Este orden del tiempo es homogéneo, congruente, reflexivo, irreflexivo, asimétrico, simétrico y transitivo a la vez. Además, el tiempo es monodimensional, tiene un sólo sentido y es irreversible. Pero no hay necesariamente simultaneidad entre el pasado, el presente o el futuro en un lugar y las correspondientes divisiones del tiempo en otros lugares. Por último, tenemos que el nexo universal entre todos los lapsos del tiempo atómico se manifiesta destacadamente en las propiedades de monocronismo, heterocronismo, policronismo, homocronismo, cinecronismo, isocronismo y anisocronismo de las partículas microcósmicas. De esta manera, hemos indicado los rasgos peculiares de la categoría de tiempo en la física atómica, como elementos para la explicación objetiva y

$43 \mathrm{Si}$ el tiempo fuese meramente subjetivo y los procesos atómicos existiesen atemporalmente, los físicos ya hubiesen prescindido del tiempo en sus explicaciones de la realidad y habrian podido ignorarlo por completo en sus concepciones. 
racional de las formas temporales de existencia y, a la vez, como base para la integración filosófica de los nuevos conocimientos adquiridos acerca de este concepto fundamental. Entonces, sólo nos falta hacer explícitamente la salvedad de que nuestra construcción es sencillamente y por entero una hipótesis sobre la categoría de tiempo en el dominio de los procesos microcósmicos que, como toda hipótesis, implica la condición ineludible de ser sometida a la prueba decisiva de su verificación en el experimento.

Eut de Gortari 